\title{
NEW BURST ASSEMBLY AND SCHEDULING TECHNIQUE FOR OPTICAL BURST SWITCHING NETWORKS
}

\author{
${ }^{1}$ V. Kavitha and ${ }^{2}$ V. Palanisamy \\ ${ }^{1}$ Department of Electronics and Communication Engineering, \\ M.Kumarasamy College of Engineering, Karur, Tamil Nadu, India \\ ${ }^{2}$ Department of Electronics and Communication Engineering, \\ Info Institute of Engineering, Coimbatore, Tamil Nadu, India
}

Received 2013-05-24, Revised 2013-06-25; Accepted 2013-07-06

\begin{abstract}
The Optical Burst Switching is a new switching technology that efficiently utilizes the bandwidth in the optical layer. The key areas to be concentrated in Optical Burst Switching (OBS) networks are the burst assembly and burst scheduling i.e., assignment of wavelengths to the incoming bursts. This study presents a New Burst Assembly and Scheduling (NBAS) technique in a simultaneous multipath transmission for burst loss recovery in OBS networks. A Redundant Burst Segmentation (RBS) is used for the burst assembly. In our proposed algorithm, the proper policy is selected based on the priority of each burst, the length of each burst and the length of the redundant data. After assembling the burst, the burst scheduler algorithm schedules the burst. This algorithm is responsible for the output wavelength and contention-free fiber delay lines to switch every burst payload to its target output fiber. The OBS network simulator (nOBS) patch in NS-2 is used to simulate our network. NBAS technique improves the quality of service constraints like delivery ratio, delay compared to RBS technique. From the simulation results, it is shown that our proposed technique New Burst Assembly and Scheduling (NBAS) optimizes the delay and overhead through the best burst assembly and scheduling thereby increasing the delivery ratio.
\end{abstract}

Keywords: OBS Networks, Burst Assembly, Redundant Burst Segmentation, Redundant Data, Burst Header Packet, Burst Scheduling, Forward Error Correction, CBR Delay, Delivery Ratio

\section{INTRODUCTION}

\subsection{OBS Networks}

There has been a solid sound growth in internet traffic and wavelength division multiplexing for several years in the past. The Optical Burst Switching (OBS) is considered as a new technology in switching. Using the OBS, the bandwidth is efficiently utilized in the optical layer. In OBS networks, packets are assembled into data bursts at the network ingress nodes and disassembled back into packets at the network egress nodes (Fernandez et al., 2013). The low cost all optical deployment of future ultrabroadband networks is through optical switching.
In OBS networks, the packets are arriving at an OBS ingress node and those packets are designated for the same egress OBS node. The aggregated packets belong to the same QoS class. At intermediate nodes, instead of sending the data within the optical signal, the whole burst is transparently switched according to the directives contained within a control packet proceeding the burst. At the egress node, the burst is subsequently de-aggregated and forwarded electronically (Cameron et al., 2005).

Contention between bursts causes high blocking probabilities and subsequent high loss rates even for moderate network utilizations because of lack of buffers

\footnotetext{
Corresponding Author: V. Kavitha, Department of Electronics and Communication Engineering,

M. Kumarasamy College of Engineering, Karur, Tamil Nadu, India
} 
within the network. Currently, most traffic on the internet is sent using control protocols that retransmit the lost data. To efficiently transmit bursts of IP data over WDM networks, the OBS is used. In an OBS network, a WDM link consists of multiple data channels to transmit the payload (data bursts) and one or more dedicated control channel to transmit the corresponding Burst Header Packets (BHP). In order to configure the switches along with the burst's route, the BHP is transmitted ahead of the burst. The burst follows the header without waiting for an acknowledgment for the connection establishment. The header and the data burst are separated by an offset time at the source as well as the subsequent intermediate nodes.

In order to configure the switches along with the burst's route, a control header is transmitted ahead of the burst. In a Delayed Reservation (DR) signaling scheme, the burst follows an out-of-band control header after some offset time without waiting for an acknowledgment for the connection establishment. While the burst is buffered electronically at the source, the offset time allows the header to be processed at each intermediate node (Wang et al., 2005). No fiber delay lines are necessary at the intermediate nodes to delay the burst while the header is being processed. The control message may also specify the duration of the burst in order to let a node know when it may reconfigure its switch for the next burst (Li et al., 2005; Vokkarane, 2007).

\subsection{Burst Assembly Techniques}

Burst assembly is the most important concern in OBS networks. Aggregating and assembling input packets into a burst at edge node is called Burst assembly. The most common burst assembly techniques are timer-based and threshold-based. A burst is created and sent to the optical network at periodic time interval is called timer-based burst assembly approach. A threshold is a limit on the number of packets contained in each burst before it is sent into the optical network. Hence, the network has fixed-size input bursts (Garg, 2013).

The burst assembling and scheduling policies have to take an account of the number of classes of packets and the number of burst priorities supported in the core to provide QoS support in the optical backbone. A burst may contain packets of a particular class or a combination of packets of different classes. Existing burst assembling schemes assemble bursts with packets of the same class. When the number of packet classes is higher than the number of burst priorities, the composite burst assembly technique can provide service differentiation.
During the burst assembly, the highest-class packets are placed at the head of the burst, while lower-class packets are placed away from the head of the burst in decreasing order of their packet class. This ordering works well with the segmentation-based contention resolution policy since the tail of the original burst which has primarily the lower-class packets will be dropped during the contention. In such a scheme, the lower-class packets are dropped for the benefit of the higher-class packets; thus, the tail of a burst effectively has lower priority than the head of a burst (Vokkarane and Jue, 2005).

\subsection{Burst Scheduling Techniques}

Once a burst is created, it must be sent to the OBS core. The process of sending the burst into the core is called the burst scheduling. The scheduling must meet the constraints of each class like loss, delay and bandwidth constraint. Burst scheduling for supporting QoS in OBS networks is different from traditional IP scheduling disciplines. In IP, each core node stores the packets in prioritized buffers and schedules them.

There are many burst scheduling principles such as First-Come First- Served (FCFS), Priority Queuing (PQ), Weighted Round Robin (WRR) and Waiting Time Priority (WTP). At First-Come First-Served (FCFS), bursts are served in the same order that they are created. In Priority Queuing (PQ), a burst is scheduled to an output port only if all burst queues of the higher priority are empty. In Weighted Round Robin (WRR), each prioritized burst queue is served in a round-robin order. In each round, the number of bursts is sent according to the weight assigned by the policy. In Waiting Time Priority (WTP), the priority of a burst is calculated by its waiting time and the priority increases with waiting time. The scheduler chooses the burst which has the largest priority at the head of the queue and sends this burst into the core (Tachibana and Kasahara, 2006). The mapping between burst priorities and burst types is an important issue in OBS networks to guarantee QoS of packet class.

The important design considerations when defining the burst types and scheduling policies are packet loss probability, delay constraints and bandwidth guarantees. The packet loss probability can be reduced by specifying an appropriate threshold.

\subsection{Previous Work}

In our previous work (Kavitha and Palanisamy, 2012), a simultaneous multi-path transmission along 
with path interleaving was proposed for reducing burst contention and also for burst loss recovery. When burst loss occurs continuously, the Forward Error Correction (FEC) encoding technique is combined with path interleaving which can be used to recover the continuous lost bursts. The path interleaving mechanism determines the block length and the number of source packets and redundant packets are fixed. Based on the redundant blocks, the continuous lost bursts can be recovered at the receiver.

The proposed technique doesn't consider the burst assembly which affects the performance improvement of the network. Discussion about how the burst scheduling is implemented in the proposed technique is not presented.

Our previous work has been extended with a new burst assembly and scheduling technique in simultaneous multipath transmission for burst loss recovery in Optical Burst Switching (OBS) networks.

\subsection{Related Works}

Pavon-Marino et al. (2009) have proposed Parallel Iterative Optical Burst Scheduler (PI-OBS) for OBS Networks. In PI-OBS, all the headers received during a given time window are jointly processed to optimize the delay and output wavelength allocation using void filling techniques and allowing traffic differentiation. The PIOBS is the first proposal of a parallel-iterative scheduler for OBS switches. In contrast to conventional greedy approaches, all the headers received in a given time window are jointly processed. The PI-OBS scheduler has been designed to allow parallel electronic implementation similar to the ones in VOQ schedulers with a deterministic response time.

Papazoglou et al. (2009) have proposed two new techniques. The first proposed technique is based on a triangular estimate that defines a "drop zone"; bursts that fall in this area are considered to have a very low probability of finding a suitable wavelength and as such, no effort is made to schedule them. According to the second approach, the drop zone is defined dynamically based on the burst drop history. The proposed algorithms will reduce the number of channels or void checks and thus the overall scheduling complexity.

Cao et al. (2009) have proposed novel algorithms for batch scheduling in OBS networks with different optimization criteria. The algorithms effectively consider the strong correlations among the multiple bursts and employ the proposed interval graphs and min-cost circular flow techniques to achieve optimized network performance in terms of data loss rate in the network.

Barradas and Medeiros (2010) have proposed four path selection strategies to prevent congestion. The first two addressed contentions in networks that assume total wavelength conversion capability including the streamline effect in a relaxed way. The next two strategies satisfied the wavelength continuity constraint without wavelength conversion and the streamline effect is strictly applied. The strategies also assume two different approaches, first considers the final path which is chosen from a set of $\mathrm{K}$ different eligible paths previously calculated and in second one, the final path is entirely determined by the algorithm.

Charbonneau et al. (2012) have proposed Forward Segment Redundancy (FSR), a proactive technique to prevent data loss during random contentions in the optical core. With FSR, redundant TCP segments are appended to each burst at the edge and Redundant Burst Segmentation (RBS) is implemented in the core so that when a contention occurs, primarily redundant data is dropped. They develop an analytical throughput model for TCP over OBS with FSR. FSR is found to improve TCP's performance by an order of magnitude at high loads and by over two times at lower loads.

\section{MATERIALS AND METHODS}

\subsection{Proposed Work}

In the proposed method, the Redundant Burst Segmentation (RBS) is used for the burst assembly. Here, one field is added to initiate the burst assembly algorithm at the ingress node. In the algorithm out of four policies, the proper policy is selected based on the priority of each burst, the length of each burst and the length of the redundant data. Those four polices are combined Head and Tail Drop (HTD), Head Drop (HD), Tail Drop (TD) and Drop Contending burst (DC).

After assembly, the bursts are scheduled using the scheduler algorithm. This algorithm is responsible for output wavelength and contention-free Fiber Delay Lines (FDL) to switch every burst payload to its target output fiber. Using this, the gap between the two consecutive bursts in an output wavelength can be avoided. The algorithm is composed of a set of delay cycles and each delay cycle consists of a sequence of 4 steps named Request, Grant, Accept and Update. 

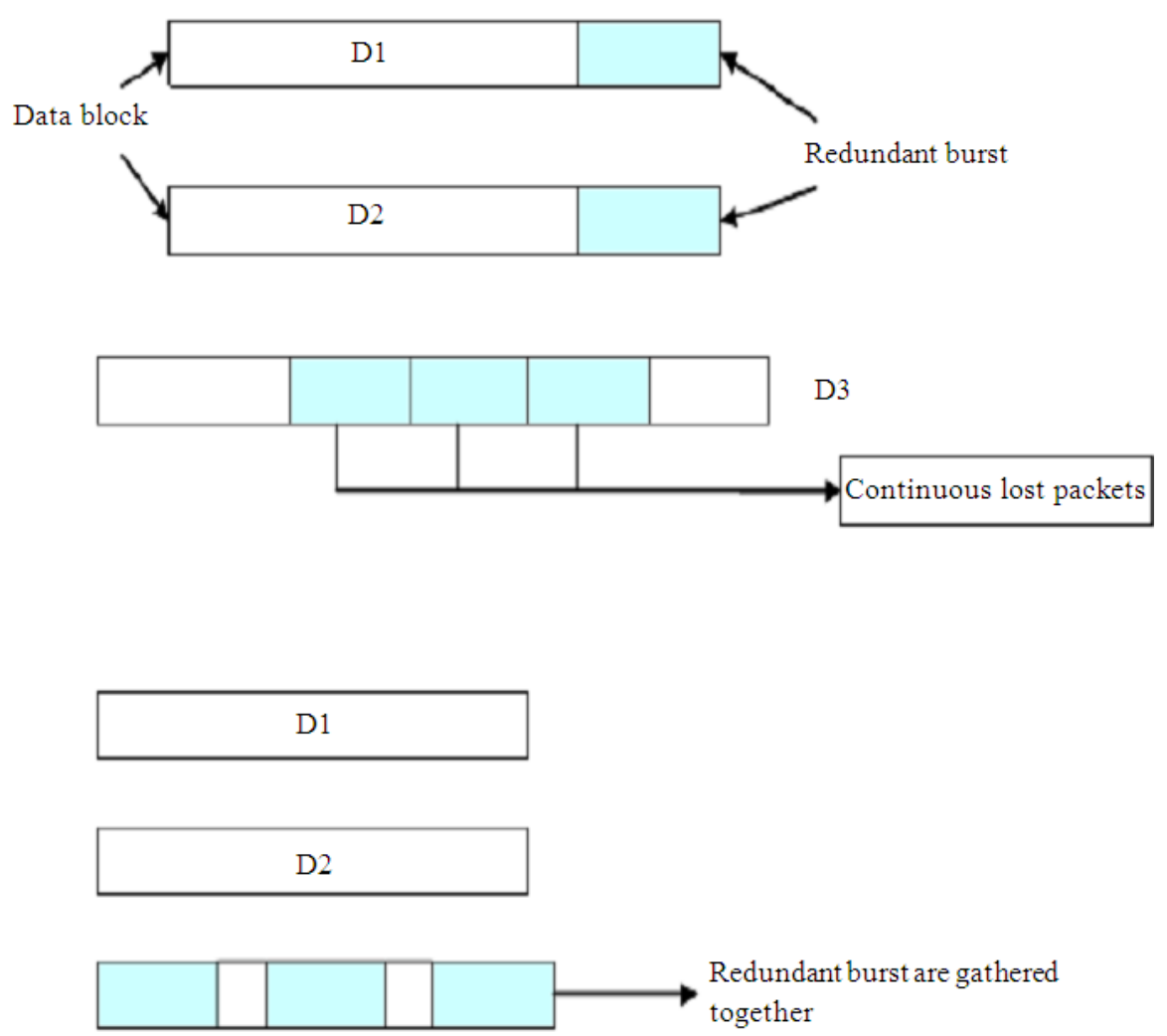

Fig. 1. Redundant Burst generation

In FEC based burst recovery technique, initially Reed-Solomon codes are considered for the FEC processing. The $(\mathrm{n}, \mathrm{k}) \mathrm{RS}$ codes include $\mathrm{n}$ byte length code words, with $\mathrm{k}$ out of $\mathrm{n}$-bytes as the original data and the remaining (n-k) bytes as the redundant data:

$$
2 \mathrm{t}+\mathrm{e}<\mathrm{n}-\mathrm{k}
$$

Equation 1 denotes the (n, k) RS code which can correct $t$ errors and e errors occurring in one n-byte code word. Here (n-k) bytes data loss can be recovered when no error occurs.

It is considered that an application data is split into $\eta$ blocks with the same size. Each data block generates a burst $\eta$ which is transmitted to the destination node. The size of the data block is $\mathrm{S}$ bytes. The parameters of RS code are chosen so that:

$$
\begin{gathered}
\mathrm{n}=(\eta+1) \lambda \text { and } \\
\mathrm{k}=\eta \lambda
\end{gathered}
$$

where, $\lambda$ is a constant value.
A new burst is constructed with redundant data of the other bursts. When one of the $\eta+1$ burst is lost transmission, it can be recovered until the destination node receives the remaining $\eta$ bursts successfully.

Figure1 shows three data blocks D1, D2 and D3 with redundant data in it. The redundant blocks in D1 and D2 are separated to form a redundant burst. FEC encoding is performed at the source node with $((\eta+1) \lambda, \eta \lambda)$ RS codes. The encoding scheme collects $\lambda$ byte data blocks from each of the bursts. The $\eta \lambda$ byte data are collected from the $\lambda$ byte data blocks. By combining these redundant blocks, a redundant burst is constructed.

$[S / \lambda]$ encoding operations need to be performed for each data burst which has S-bytes and it generates $\lambda$ redundant data. Original burst $\eta$ and the generated redundant burst are transmitted to their destination node. At an intermediate node, when one of the $\eta+1$ burst is lost, FEC decoding algorithm recovers the lost burst at the destination node. Since the lost original burst can be recovered from the other original burst and redundant data, the transmission of the two original bursts succeeds. 
The FEC decoding time can be calculated using $\mathrm{P}$ number of FEC processors and $\mathrm{W}$ bps FEC processing speed for each FEC processor. The FEC decoding time $(\delta)$ is given by Equation 2:

$$
\delta=\frac{8(\eta+1) S}{P W}
$$

The burst D3 has continuous lost data blocks and thus it has to be recovered using the FEC encoding technique combined with path interleaving.

\subsection{Burst Assembly Algorithm}

The Redundant Burst Segmentation (RBS) is used for the burst assembly to reduce the losses due to random contentions at core nodes. In RBS, the burst is divided based on the redundant data. Priorities are allotted to the burst when it is divided. The burst with redundant data is considered as a low priority $\operatorname{LP}\left(\mathrm{P}_{1}\right)$. The burst which does not contain redundant data is considered as high priority $\mathrm{HP}\left(\mathrm{P}_{0}\right)$.

\subsubsection{Modified Structure of Burst Header Packet}

Once the burst is ready before sending it, a BHP is created. A BHP contains the routing information and information about the data burst Fig. 2. Each burst consists of a number of segments which has a header and a payload. A BHP is sent and processed electronically at each core node to set up the optical switching fabric. The BHP specifies the length of the redundant data, guard bits, payload type, segment ID, segment length and checksum. The BHP is sent before the burst by an offset time. One field is added to initiate the burst assembly algorithm at the ingress node. That field contains the length of the redundant data. In the algorithm, the proper policy is selected out of the four policies like combined Head and Tail Drop (HTD), Head Drop (HD), Tail Drop (TD) and Drop Contending burst (DC):

- Combined Head and Tail Drop (HTD): A portion of each burst is dropped. The part of the head of the contending burst and part of the tail of the original burst is dropped. The dropping part is determined when the contention occurs.

- Head Drop (HD: The head of the contending burst is dropped so that there is no longer contention.

- Tail Drop (TD): There is no longer contention because the tail of the original burst will be dropped.
- Drop Contending burst (DC): The original burst is scheduled and the entire contending burst is dropped

The proper policy is selected based on the length of the redundant data in the contending burst, the length of the original burst, length of the overlapped data in the burst and length of the contending burst. follows:

For selecting the policy, the proposed algorithm is as

$$
\begin{aligned}
& \text { 1. Start } \\
& \text { 2. Define } \mathrm{P}_{1} \text { : the length of the redundant data in } \\
& \text { the contending burst. } \\
& \text { 3. } \mathrm{P}_{0} \text { : the length of the original burst } \\
& \text { 4. LB: length of the overlapped data in the burst } \\
& \text { 5. LC: length of the contending burst; } \\
& 6 . \\
& \text { 7. If }\left(\mathrm{P}_{0}>0\right) \\
& \text { 8. }\{ \\
& \text { 9. If }\left(\left(\mathrm{P}_{1}>0\right) \text { and }(\mathrm{LC}>\mathrm{LB})\right) \\
& \text { 10. } \quad \text { Policy }=\text { HTD at midpoint; } \\
& \text { 11. If } \left.\left(\left(\mathrm{P}_{1}>0\right) \text { and } \mathrm{LC}<\mathrm{LB}\right)\right) \\
& \text { 12. } \quad \text { Policy }=\text { HTD at Tail; } \\
& \text { 13. If }\left(\mathrm{P}_{1}>0\right) \\
& 14 . \\
& 15 . \\
& \text { 16. If }\left(\mathrm{P}_{1}>0\right) \\
& \text { 17. } \quad \text { \} } \\
& \text { 18. If }\left(\mathrm{P}_{1}=0 \text { and } \mathrm{P}_{0}=0\right) \\
& 19 . \\
& 20 . \\
& \text { Policy = HTD at midpoint; }
\end{aligned}
$$

In the algorithm, the length of the original burst has high priority and the length of the redundant data in the contending burst has low priority. The HTD midpoint is the point at which the part of the head of the contending burst and part of the tail of the original burst is dropped. The midpoint of the burst is calculated using the following Equation 3:

$$
\text { midpoint }=\mathrm{LAT}_{\mathrm{i}}-\left[\frac{\mathrm{LB}}{2}\right]
$$

$\mathrm{LAT}_{\mathrm{i}}$ is the latest available time for wavelength $\mathrm{i}$ and LB is the length of the overlapped data in the burst. At HTD, the tail of the original burst is dropped.

\subsection{Burst Scheduling Algorithm}

The burst scheduler algorithm is a parallel iterative algorithm. Let us assume the response time of the upper 
bound is $\mathrm{T}_{\mathrm{ru}} \mu \mathrm{s}$ and the algorithm is executed for every $\mathrm{T}_{\mathrm{i}} \mu \mathrm{s}$. The algorithm must start after the previous execution is finished and it must follow $\mathrm{T}_{\mathrm{i}}>\mathrm{T}_{\mathrm{ru}}$. The algorithm starting time is $\mathrm{T}=\mathrm{T}_{0}$. The starting time is responsible for processing the burst headers asynchronously and the time interval is $\left[\left(\mathrm{T}_{0}-\mathrm{T}_{\mathrm{i}}\right), \mathrm{T}_{\mathrm{o}}\right]$ which is called the header arrival time window of the execution. After the execution of the algorithm, the scheduling decisions made for all the processed headers are stored in the system.

Once the burst is assembled, it is scheduled by the burst scheduler algorithm. After the burst assembly, the bursts are entered into the input module. In input module, the Horizon time block is used to schedule the bursts. In each input module, information about the burst allocation like burst offset, burst length and QoS class are to be updated during the algorithm iterations Fig. 3.

The horizon time block is assigned to each and every burst. During the horizon time, the burst enters the interconnection matrix. The interconnection matrix consists of sequence of delay cycles and each delay cycle consists of a sequence of four steps which are request, grant, accept and update. Using this, the gap is avoided between the two consecutive bursts in an output wavelength. The resources are allocated to the burst based on the delay cycle (i.e., calculate using the Equation 7). The decision should be made in the interconnection matrix only. The allocation can be done in output module. Each output module contains four registers $R_{1}, R_{2}, R_{3}$ and $R_{4}$. These registers store the occupation of the output wavelength $w$ along time, grant pointer and direction of grant pointer. The grant pointer grants the resource to the bursts.

\subsubsection{Input Module}

In the input module, the horizon time block $\mathrm{H}=$ $0, \ldots, h-1)$ is considered in the consecutive intervals of duration $T_{i}$, at which the future payload arrivals are made. Each input wavelength is $\mathrm{IW}=0, \ldots, \mathrm{i}-1$ and input fiber is IF $=0, . ., \mathrm{i}-1$. One input module IM exists per each horizon time block and an input module contains the information about a payload whose first bit arrives the OSF (Optical switching fabric transparently switches optical bursts from input ports to output ports) through input fiber IF, at input wavelength IW, within the time interval. The time interval is $\left[\left(\mathrm{T}_{0}-\mathrm{T}_{\mathrm{k}}\right)\left(\mathrm{T}_{0}-\mathrm{T}_{\mathrm{j}}\right)\right]$. Here, $\mathrm{T}_{\mathrm{k}}$ and $\mathrm{T}_{\mathrm{j}}$ are:

$\mathrm{T}_{\mathrm{k}}=\mathrm{T}_{\mathrm{i}}+\mathrm{T}_{\mathrm{wt}}+\mathrm{hT}_{\mathrm{i}}$
$\mathrm{Tj}=\mathrm{T} j+\mathrm{Twt}+(\mathrm{h}+1) \mathrm{Tj}$

In Equation 4, $\mathrm{T}_{\mathrm{i}}$ is the periodic time that an algorithm is executed for every periodic time; $\mathrm{T}_{\mathrm{wt}}$ is the worst case time that is spanning between the instant of header reception and the moment at which a path is ready for the payload. It depends on the three parameter $T_{i}, T_{A}$ and $T_{0} . T_{i}$ is the time of the header arrival for algorithm execution in the worst case; $\mathrm{T}_{\mathrm{A}}$ is the algorithm response time and $\mathrm{T}_{\mathrm{o}}$ is the optical components of the OSF Equation 6:

$\mathrm{T}_{\mathrm{wt}}=\mathrm{T}_{\mathrm{i}}+\mathrm{T}_{\mathrm{A}}+\mathrm{T}_{\mathrm{o}}$

The worst case time is always less than the minimum offset time and delay. The minimum offset time between the burst header and the burst payload is $\mathrm{T}_{\mathrm{m}}$. The Delay (D) is the time duration of header arrival times at the input port:

$\mathrm{T}_{\mathrm{m}}+\mathrm{D} \geq \mathrm{T}_{\mathrm{wt}}$

The Equation 5 must be applied because the sum of the minimum offset time and delay always greater than the worst case time. The time $T_{0}-T_{i}+T_{w t}$ is the earliest time of arrival of a payload whose header is received at the start of the current header arrival time window, $t=\mathrm{t}_{0^{-}}$ $\mathrm{T}_{\mathrm{i}}$. The difference between the maximum and minimum offset time allowed in the system decides the number of time Horizons $(\mathrm{H})$.

Information about the burst allocation has to be updated during the iterations of the algorithm, burst offset, burst length and QoS class are stored in each input module.

\subsubsection{Output Module}

The output module exists for each output fiber $\mathrm{OF}=$ $0, \ldots, \mathrm{i}-1$ and output wavelength $\mathrm{OW}=0, \ldots \ldots, \mathrm{i}-1$. Each output module contains four registers $\mathrm{R}_{1}, \mathrm{R}_{2}, \mathrm{R}_{3}$ and $\mathrm{R}_{4}$. $\mathrm{R}_{1}$ and $\mathrm{R}_{2}$ stores the occupation of the output wavelength $w$ along time. $\mathbf{R}_{3}$ stores the grant pointer and $\mathbf{R}_{4}$ stores the direction of the grant pointer.

Step-by-step process of Burst scheduler algorithm:

- Let us assume algorithm starts at time $\mathrm{T}=\mathrm{T}_{0}$.

- Input modules are $\mathrm{H}=0, . ., \mathrm{h}-1, \mathrm{IF}=0, \ldots, \mathrm{i}-1, \mathrm{IW}=$ $0, \ldots, \mathrm{i}-1$. 


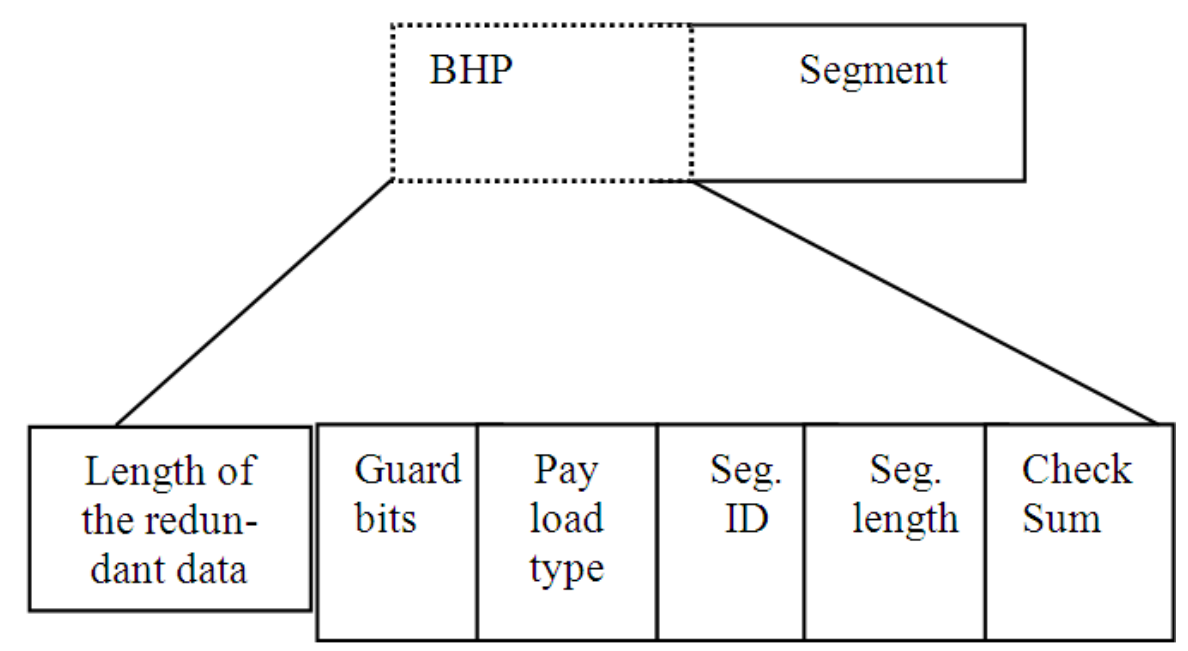

Fig. 2. Burst header packet format

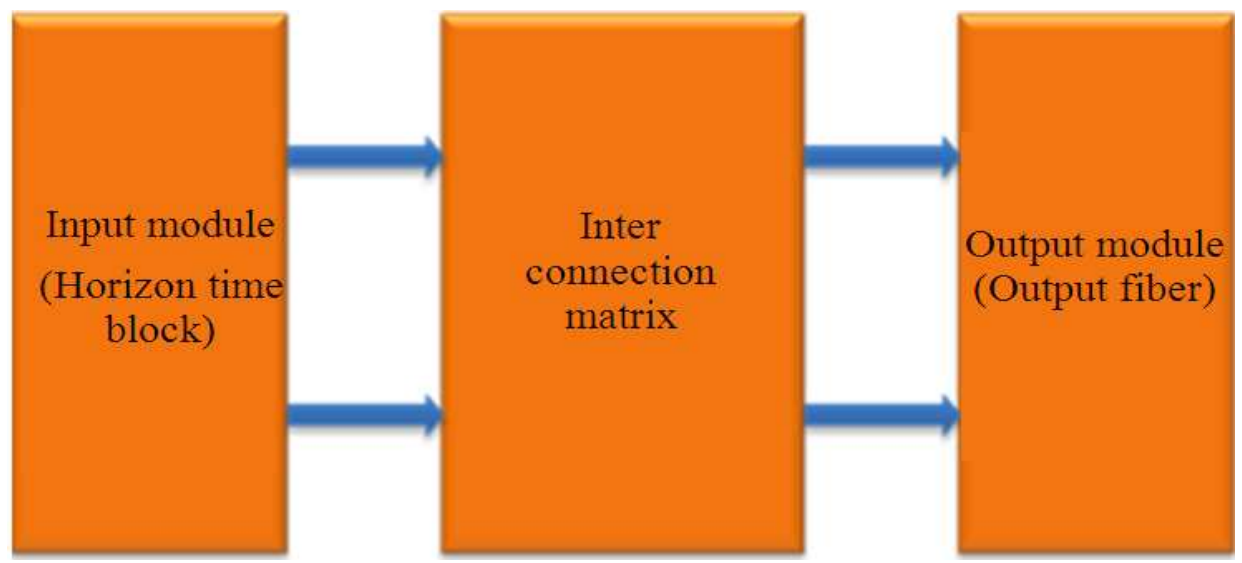

Fig. 3. Burst scheduler architecture

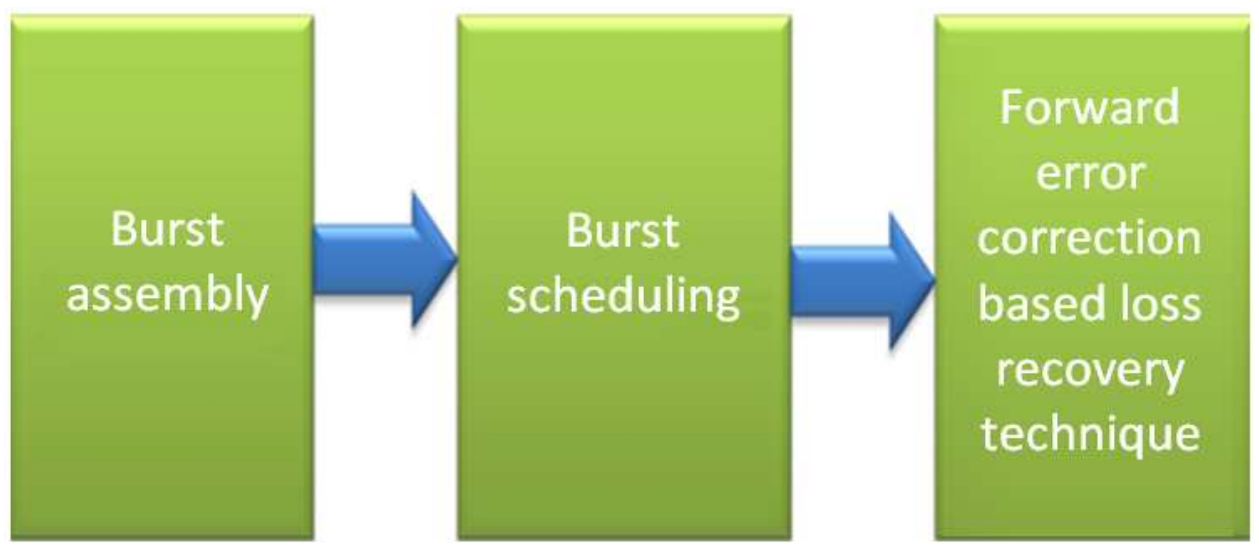

Fig. 4. Block diagram of New Burst Assembly and scheduling technique 


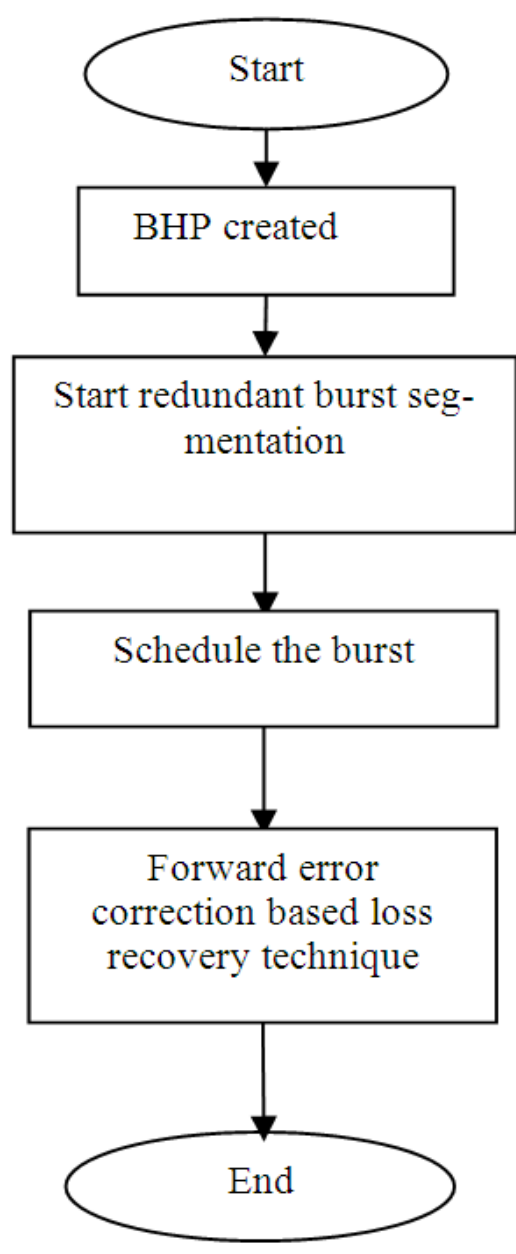

Fig. 5. Flow chart for New Burst Assembly and Scheduling technique

- Input contains the information about the burst headers, at what time they arrived

- The $\mathrm{R}_{1}, \mathrm{R}_{2}$ contains the same information and $\mathrm{R}_{1}$ will act as a backup copy of $\mathrm{R}_{2}$ during algorithm execution

- The algorithm is composed of iterations and each iteration is composed of a sequence of delay cycles.

- Each delay cycle consists of a sequence of 4 steps: (i) request, (ii) grant, (iii) accept and (iv) update

- Request step: The input module $\mathrm{i}$ with payload to output fiber $f$, a request signal is sent to the output modules associated with all output of the target output fiber. After the request signal, information about the payload arrival time, payload duration and payload QoS is also through the signaling interconnection matrix
- Grant step: The signals can be scanned and received from the request. The burst that doesn't overlap with existing burst schedule in previous execution, not allocated bursts in delay cycles of the same algorithm iteration are eligible for the grant and then grant is sent to that burst. Using the interconnection matrix, the grant signal is transmitted

- Accept step: Once the grant signal is obtained and it holds more than one grant, the one with the lowest index. Then an accept signal is sent to the associate output module. Once the accept signal is sent, the input module does not enter into play for future delay cycles in the same iterations and only the input modules which sent a request can receive a grant.

- Update step: The internal register $\mathrm{R}_{2}$ is updated with new accept signal information, so that future in 
different delays of the same iteration do not overlap with the accepted allocation

- Once the iteration is finished, all the performed assignments are erased

- At the end of the last iteration, the allocations by the input modules are considered final

This algorithm is responsible for output wavelength and contention-free Fiber Delay Lines (FDL) to switch every burst payload to its target output fiber. Using this, the gap between the two consecutive bursts in an output wavelength can be avoided.

\subsection{New Burst Assembly and Scheduling Technique (NBAS)}

In New Burst Assembly and Scheduling technique, a BHP is created to send the burst. The BHP contains the routing information and information about the data burst. The burst is assembled using any one of policy in the assembly algorithm (2.2).

From Fig. 4 and 5, it is noted that the assembled burst is scheduled by the burst scheduler. If there is any error during the burst transmission, the Forward Error Correction based loss recovery technique is used to recover the bursts without loss (Kavitha and Palanisamy, 2012).

\section{RESULTS}

\subsection{Simulation Model and Parameters}

We examine the performance of our New Burst Assembly and Scheduling Technique (NBAS) with an extensive simulation study based upon the NS-2 network simulator. The OBS network simulator (nOBS) patch in NS-2 is used to simulate our network.

In simulation, an exponential traffic model is used to generate bursty traffic in which 5 traffic flows are setup between two edge routers. In all the simulation environments, we compare the results of the proposed NBAS technique with Redundant Burst Segmentation (RBS) technique (Charbonneau et al., 2012).

\subsection{Performance Metrics and Analysis}

The following metrics are measured in all the simulation experiments:

- Burst Delay: It is the average end to end delay between the sender and the receiver

- CBR Delay: It is delay occurred during Constant Bit Rate traffic

- Burst Received: It is the number of optical bursts at the edge receiver
- Bytes Received: It is the number of bytes received successfully

- Delivery Ratio: It is the ratio between the number of bursts received and the numbers of bursts sent

\section{DISCUSSION}

The simulation results show that NBAS technique outperforms RBS technique in terms of QoS parameters. The parameters are noted when the load is varied as 10 , 20, 30, 40 and $50 \mathrm{Mb}$.

Load is classified into two types as low load traffic with a load of $10 \mathrm{Mb}$ and high load traffic with a load of $50 \mathrm{Mb}$.

The simulation results are averaged over 5 runs. From Fig. 6, it is observed that the increase in load causes more data traffic resulting in increased delay. NBAS has an average of $18.8 \%$ lesser burst delay than RBS.

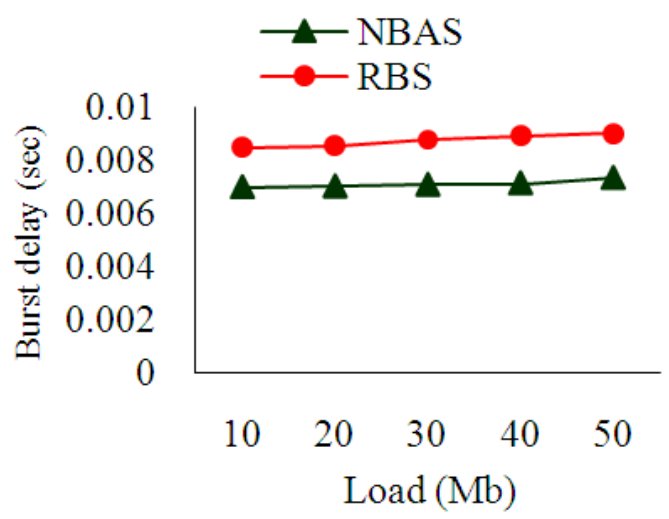

Fig. 6. Load Vs Burst delay

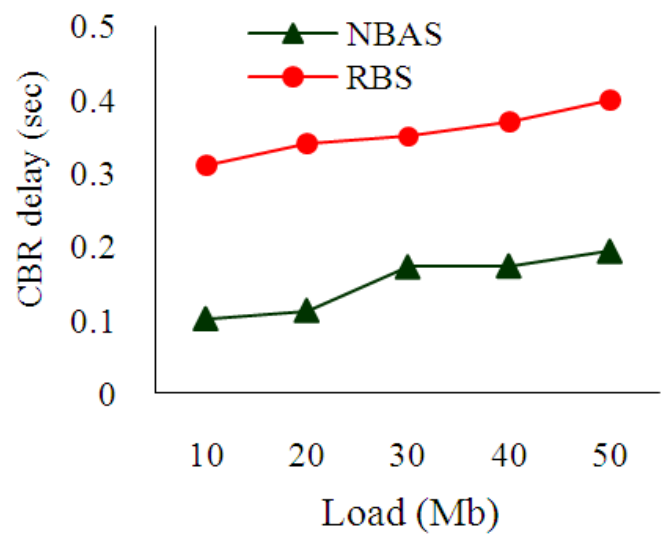

Fig. 7. Load Vs CBR-delay 


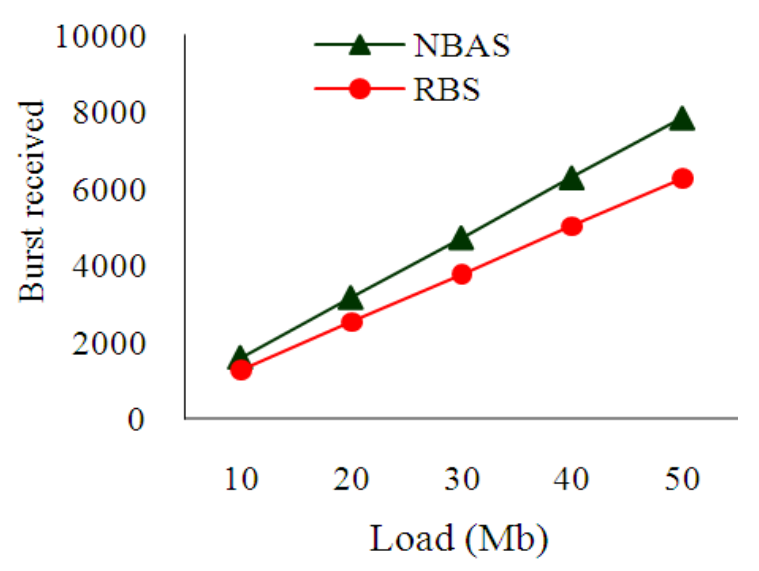

Fig. 8. Load Vs burst received

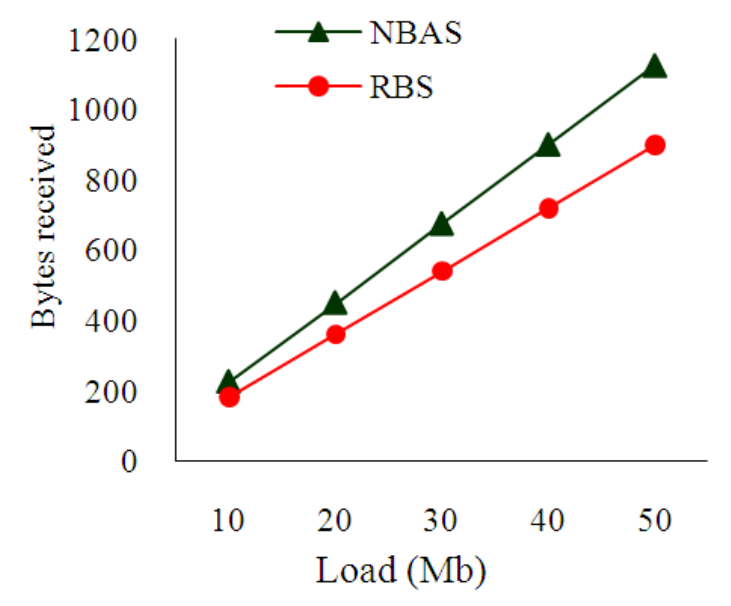

Fig. 9. Load Vs bytes received

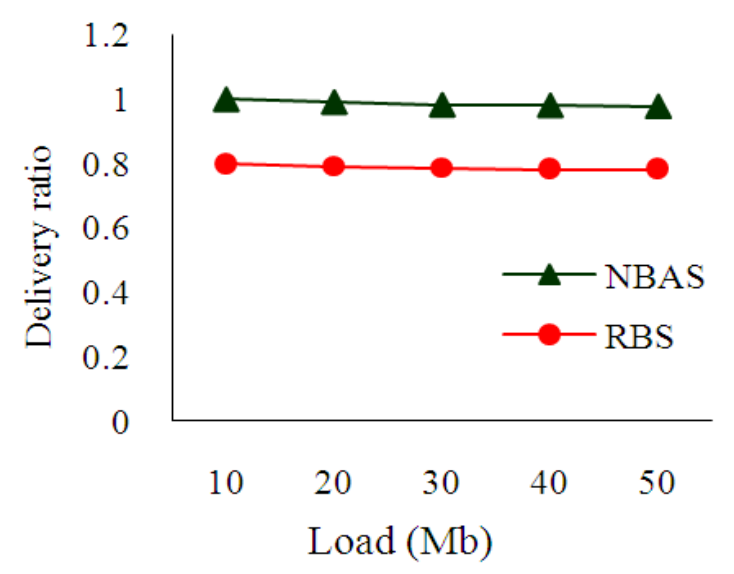

Fig. 10. Load vs delivery ratio
Figure 7 shows the results of CBR delay for various load conditions. From the results, we can see that NBAS techniques has an average $57.7 \%$ lower delay than the other scheme RBS.

Figure 8 and 9 show the burst received and bytes received at the destination with NBAS technique are higher than RBS i.e. an average of $19.6 \%$ and $20 \%$ high respectively. It clearly indicates the burst loss is recovered even at higher load.

Figure 10 shows the results of burst delivery ratio for the varying load scenario. Clearly, our NBAS technique achieves an average of $20 \%$ better delivery ratio than RBS.

\section{CONCLUSION}

In this In this study, we have proposed a New Burst Assembly and Scheduling technique in simultaneous multi-path transmission for burst loss recovery in Optical Burst Switching (OBS) networks. First, the Redundant Burst Segmentation (RBS) is used for the burst assembly. Here, one field is added to initiate the burst assembly algorithm at the ingress node. After assembly of burst, it is scheduled by the scheduler algorithm. This algorithm is responsible for output wavelength and contention-free fiber delay lines (FDL) to switch every burst payload to its target output fiber. It avoids the gap between the two consecutive bursts in an output wavelength. From the simulation results, it is shown that the proposed technique optimizes the Delay and Delivery Ratio.

The proposed technique can be considered as a differentiated service since it drops the bursts based on their priority. An area of future work is to find the simulation response for varying the burst size and analyze the QoS parameters.

\section{REFERENCES}

Barradas, A.L. and M.C.R. Medeiros, 2010. Pre-planned optical burst switched routing Strategies considering the streamline effect. Photon. Netw. Commun., 19: 161-169. DOI: 10.1007/s11107-009-0221-y

Cameron, C., A. Zalesky and M. Zukerman, 2005. Prioritized Deflection Routing in Optical Burst Switching Networks. IEICE Trans. Commun., E88B: 1861-1867. DOI: 10.1093/ietcom/e88-b.5.1861

Cao, X., Y. Wang and A. Zelikovsky, 2009. Scheduling bursts using interval graphs in optical burst switching networks. Proceedings of the IEEE Global Telecommunications Conference, Nov. 30-Dec. 04, IEEE Xplore Press, Honolulu, pp: 1-6. DOI: 10.1109/GLOCOM.2009.5426139 
Charbonneau, N., D. Chandran and V.M. Vokkarane, 2012. Improving TCP performance over Optical Burst-Switched (OBS) networks using forward segment redundancy. Photon. Netw. Commun., 23: 1-15. DOI: $10.1007 / \mathrm{s} 11107-011-0330-2$

Fernandez, T.F., T. Megala and N. Sreenath, 2013. Challenges, issues and research directions in optical burst switching. Int. J. of Comput. Appli. Technol. Res., 2: 131-136. DOI: 10.7753/IJCATR0202.1009

Garg, A.K., 2013. Traffic prediction based burst assembly mechanism for OBS. Int. J. Light Elect. Optics, 124: 2017-2019. DOI: 10.1016/j.ijleo.2012.06.038

Kavitha, V. and V. Palanisamy, 2012. Simultaneous Multi-Path Transmission for Burst Loss Recovery in Optical Burst Switching (OBS) Networks. Eur. J. Sci. Res., 87: 412-426.

Li, J., G. Mohan and K.C. Chua, 2005. Dynamic load balancing in IP-over-WDM optical burst switching networks. Comput. Netw., 47: 393-408. DOI: 10.1016/j.comnet.2004.07.019

Papazoglou, C., P.G. Sarigiannidis, G.I. Papadimitriou and A.S. Pomportsis, 2009. Techniques for improved scheduling in optical burst switched networks. Proceedings of the International Symposium on Autonomous Decentralized Systems, Mar. 23-25, IEEE Xplore Press, Athens, pp: 1-4. DOI: 10.1109/ISADS.2009.5207359
Pavon-Marino, P., J. Veiga-Gontan, A. OrtunoManzanera, W. Cerroni and J. Garcia-Haro, 2009. PI-OBS: A Parallel iterative optical burst scheduler for OBS networks. Proceedings of the International Conference on High Performance Switching and Routing, Jun. 22-24, IEEE Xplore Press, Paris, pp: 1-8. DOI: 10.1109/HPSR.2009.5307426

Tachibana, T. and S. Kasahara, 2006. Performance analysis of timer-based burst assembly with slotted scheduling for optical burst switching networks. Int. J. Perform. Eval., 63: 1016-1031. DOI: 10.1016/j.peva.2005.11.005

Vokkarane, V.M. and J.P. Jue, 2005. Segmentationbased nonpreemptive channel scheduling algorithms for optical burst-switched networks. J. Lightwave Technol., 23: 3125-3137. DOI: 10.1109/JLT.2005.856265

Vokkarane, V.M., 2007. Intermediate-node-initiation (INI): A generalized signaling framework for optical burst-switched networks. Opt. Switch. Network., 4: 20-32. DOI: 10.1016/j.osn.2006.09.004

Wang, J., Y. Song, S. Wang and Q. Zeng, 2005. Study on optimization of deflection routing in optical burst switched networks. Proceedings of SPIE 5625, Optical Transmission, Switching and Subsystems II Conference, Feb. 28-28, SPIE, pp: 714-721. DOI: $10.1117 / 12.575931$ 